\title{
Comparison and Research on Insulation Monitoring Methods For Marine Power System
}

\author{
Wanxuan Zhu, a , Zhiliang $\mathrm{Wu}^{2, \mathrm{~b}}$ \\ ${ }^{1}$ Marine Eng. College, Dalian Maritime Univ., Dalian, 116026, China \\ ${ }^{2}$ Marine Eng. College, Dalian Maritime Univ., Dalian, 116026, China \\ aemail:zwx19930321@163.com, bemail: wzldmu@126.com
}

Keywords: Insulation monitoring, fault phase location, dc superposition method, double frequency principle, zero sequence current amplitude comparison method

\begin{abstract}
Specific to the usual methods for insulation monitoring-dc superposition method, double frequency principle and zero sequence current amplitude comparison method, the work principle and applications of these methods were compared and analyzed, and respective characteristics were concluded. On this basis, a kind of intelligent insulation monitoring device based on S7-1200 PLC was designed. Compared to traditional megameter, this design can make the insulation data display in a digital facility, improving the accuracy of insulation data, and realizing long-range transmission of insulation data by the integrated Profinet port on PLC.
\end{abstract}

\section{Introduction}

The work mode of distribution line for marine power grid generally adopts the three-wire insulated system, the specification of the mode is that it won't cause interruption of power supply because of short-circuit tripping when there appears single phase grounding, ensuring the continuity and reliability of marine power supply. And it wouldn't influence the symmetrical relation between three line voltages, the electrical driven devices can work normally, but there exist some hidden dangers, it will increase the danger of getting an electric shock and appearing short-circuit between distributing lines ${ }^{[1]}$.

With the constant increasing of power capacity, complexity and intensity, the requirements for reliability, real-time performance, accuracy of system are higher and higher. Nowadays, the usual methods for realizing marine insulation monitoring are dc superposition method, double frequency principle and zero sequence current amplitude comparison method. There are some unique characteristics and disadvantages for these methods, this passage will compare and analyze them in detail. On this basis, utilizing megameter method to design a kind of marine intelligent insulation monitoring device, S7-1200 PLC which manufactured by siemens company is chosen to accomplish the collection and procession of insulation data and three phase voltages. The communication between PLC and Weinview touch screen is achieved by the industry Ethernet, and then PLC can transmit the processed data to interface HMI to display the insulation data in real time. The design can realize insulation on-line monitoring in real time, history alert recording, insulation fault phase location and digital display.

\section{DC superposition method}

DC superposition method is to inject dc current into power grid on line, and then judging the insulation by detecting the dc current of power grid. The working principle is similar to megameter, there is a difference in power supply, the power of megameter is to transfer, rectify line voltage, and then inserting it into power net to form a current circuit, but dc superposition method directly provide a dc power supply. As figure 1 shows, $\mathrm{E}$ is the dc power supply, $\mathrm{R}_{0}$ is a current-limiting resistor, $R_{1}$ is a detecting resistor, $R_{a}, R_{b}, R_{c}$ are the phase insulation resistor to ground, $E_{a}, E_{b}, E_{c}$ and $\mathrm{Z}_{\mathrm{a}}, \mathrm{Z}_{\mathrm{b}}, \mathrm{Z}_{\mathrm{c}}$ are the phase voltage and inner impedance of the generator respectively. 


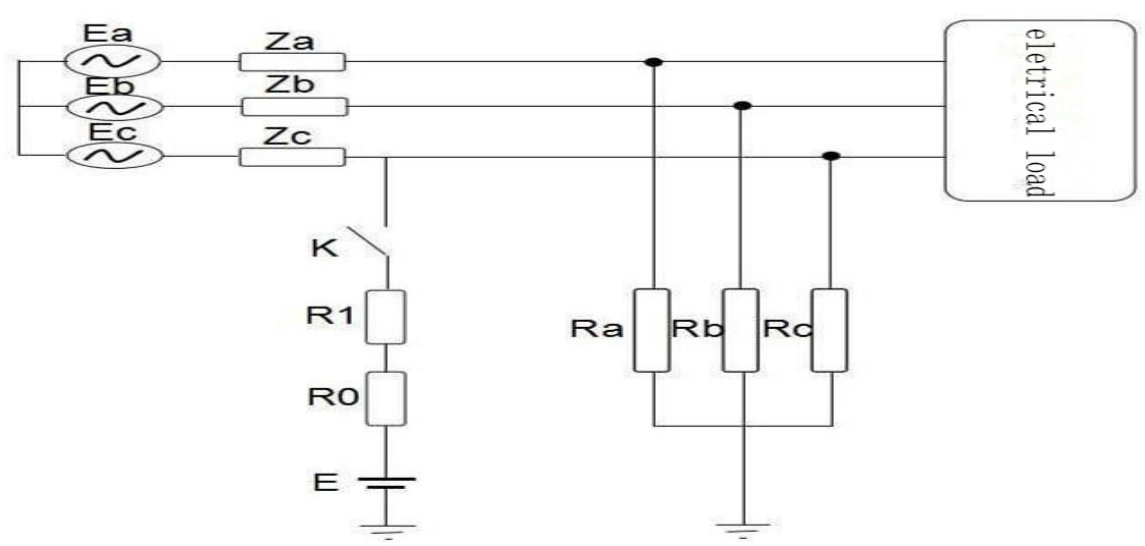

Figure 1 the schematic diagram of DC superposition method

The fundamental principle is to inject a dc current whose EMF is E between marine power gird and the hull, because the inner impedance of high-capacity generator are much less than the resistance of current-limiting resistor and detecting resistor, it can be ignored, and then the equivalent schematic diagram of insulation detecting circuit as shown in Figure2. $\mathrm{R}$ is a parallel resistance of $R_{a}, R_{b}$ and $R_{c}$, that is the required insulation data, it can be calculated by detecting the steady-state voltage $U$ (dc component) of detecting resistance. According to equivalent schematic diagram:

$$
R=\frac{E \bullet R_{1}}{U}-R_{0}-R_{1}
$$

In this formula: $\mathrm{R}_{0}$ is a current-limiting resistance, $\mathrm{R}_{1}$ is a detecting resistance

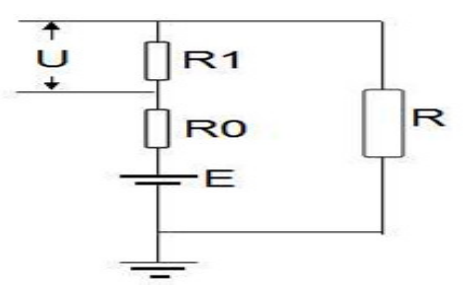

Figure 2 the equivalent schematic diagram of insulation detecting circuit

DC superposition method hardly changes the structure of power grid, it is easy to install and operate. Because the method adopts a low power, low voltage, voltage-stabilized power supply, the device is hardly influenced by AC power net, and it is easy to detect the voltage of detecting resistor, and the antijamming ability is strong ${ }^{[2][3]}$.

\section{Double frequency principle}

Double frequency principle is used to realize insulation fault locating when there appears insulation fault in power grid, it is usually used combine with zero sequence current amplitude comparison method. After insulation fault happened, firstly zero sequence current amplitude comparison method should be used to compare the amplitude of current for each branch, if the insulation fault still cannot be located, and then double frequency principle is considered to realize insulation fault location. 


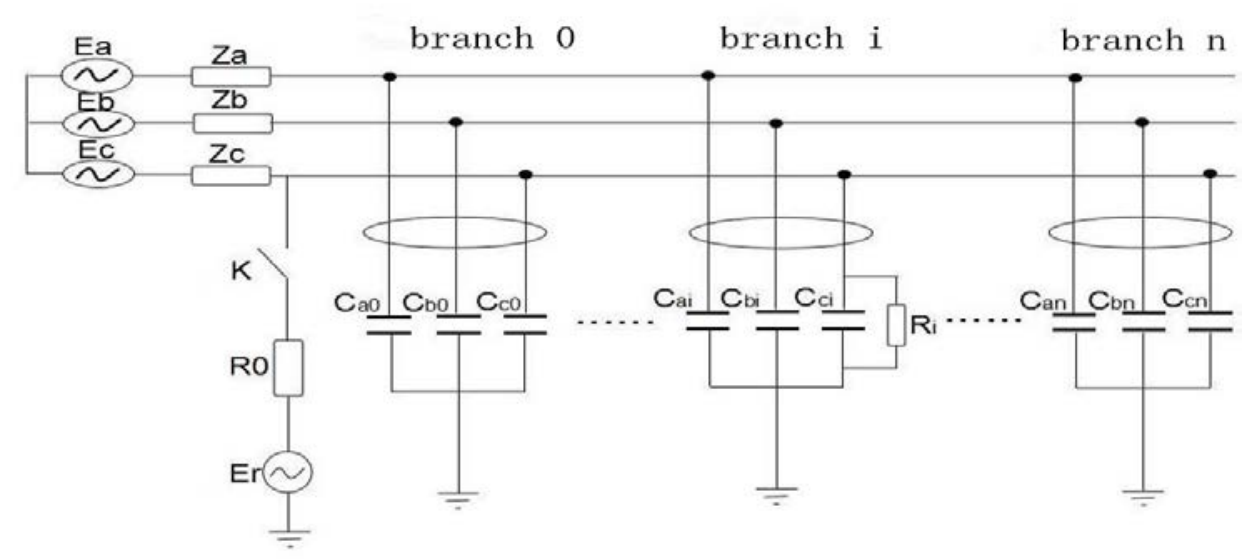

Figure 3 the schematic diagram of double frequency injection method

The schematic diagram of double frequency principle is shown as Figure 3. In this diagram, $\mathrm{E}_{\mathrm{r}} \mathrm{is}$ low frequency power supply, $\mathrm{E}_{\mathrm{a}}, \mathrm{E}_{\mathrm{b}}, \mathrm{E}_{\mathrm{c}}$ are phase voltages of generator, $\mathrm{C}_{\mathrm{a}}, \mathrm{C}_{\mathrm{bi}}, \mathrm{C}_{\mathrm{ci}}$ are cable capacitance, $\mathrm{R}_{\mathrm{i}}$ is the fault insulation resistor, the leakage current transducers are installed in each load branch. When there appears insulation fault in power net, the power supply $\mathrm{E}_{\mathrm{r}}$ will sequentially inject two frequency $\mathrm{f}_{1}$ and $\mathrm{f}_{2}$ sinusoidal current between marine power gird and the hull. Usually the inner resistance of generator can be ignored comparing to cable capacitance and insulation resistance, when the low frequency source individually act on the power grid, the equivalent schematic diagram can be drawn as Figure4.

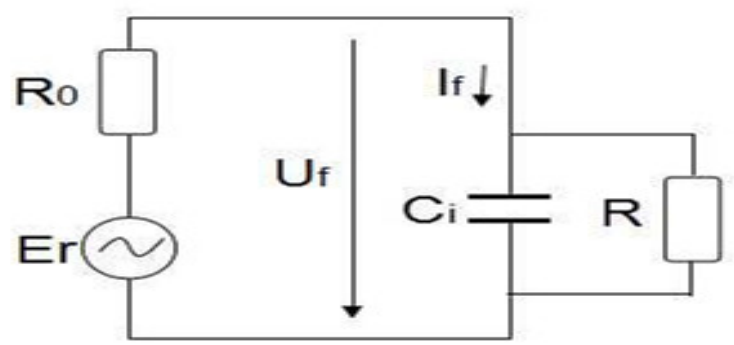

Figure4 the equivalent diagram when the source individually working

When the frequency of injected current to grid is $f_{1}$, suppose the voltage of fault branch is $U_{f 1}$, the leakage current is $I_{f 1}$. When the frequency of injected current to grid is $f_{2}$, suppose the voltage of fault branch is $\mathrm{U}_{\mathrm{f} 2}$, the leakage current is $\mathrm{I}_{\mathrm{f} 2}$. and then following equations can be gotten:

$$
\begin{aligned}
& \dot{I}_{f 1}=\dot{U}_{f 1} / R+j 2 \pi f_{1} C_{i} \dot{U}_{f 1} \\
& \dot{I}_{f 2}=\dot{U}_{f 2} / R+j 2 \pi f_{2} C_{i} \dot{U}_{f 2}
\end{aligned}
$$

So the insulation resistance of fault branch can be calculated by solving above equations:

$$
R=\frac{U_{f 1} U_{f 2} \sqrt{\mathrm{k}^{2}-1}}{\sqrt{k^{2} U_{f 1}^{2} I_{f 2}^{2}-I_{f 1}^{2} U_{f 2}^{2}}} \quad k=f_{1} / f_{2}
$$

When the insulation resistance of detecting branch is lower than set point, and then it can be confirmed as the fault branch. But in practical applications, because of the existence of power frequency current, the current detected by leakage current transducer is the sum of lower frequency current and power frequency current. it needs a filter to get the lower frequency current from the complex current, and it also need a high-precision lower frequency power supply, so it is difficult to realize ${ }^{[4]}$.

Double frequency principle can calculate the insulation resistance for each branch according to the detecting current, and it can locate the fault branch. But the structure is complex, and the control precision is required enough high for frequency converter because it need to inject different frequency current to power grid ${ }^{[5]}$. 


\section{Zero sequence current amplitude comparison method}

If there is no insulation fault, the zero sequence current of each branch is zero. But if there is insulation fault, the zero sequence current in fault branch is much bigger than that in normal branches. Zero sequence current amplitude comparison method can locate the fault branch by comparing the current amplitude of each branch.



Figure 5 the schematic diagram of zero sequence current amplitude comparison method

As figure5 shown, if there is insulation fault in branch $f$, suppose the grounding resistance is $R_{f}$, the zero sequence current of the branch ${ }^{[6]}$ :

$$
I_{0 f}=\frac{\omega\left(\mathrm{C}-\mathrm{C}_{f}\right) \mathrm{E}_{\mathrm{A}}}{\sqrt{1 / 9+\omega^{2} C^{2} R_{f}^{2}}}
$$

the zero sequence current of normal branches:

$$
I_{0 i}=\frac{\omega C_{i} E_{A}}{\sqrt{1 / 9+\omega^{2} C^{2} R_{f}^{2}}}
$$

In above two formulas: $C$ is the total phase capacitance to ground, $C_{f}$ and $C_{i}$ are the phase capacitance of fault and normal branch respectively. $\mathrm{E}_{\mathrm{A}}$ is phase voltage.

Because the total phase capacitance to ground $\mathrm{C}$ is much bigger than the capacitance of branches $\mathrm{C}_{\mathrm{f}}$ and $\mathrm{Ci}$, the amplitude of all normal branches are much less than fault branch. In the theory, a amplitude comparator is provided for all branches, then the branch whose amplitude is biggest can be confirmed as fault branch. The working principle of zero sequence current amplitude comparison method is more simple than other methods, it performs perfectly for single phase grounding, but if the total phase capacitance to ground is not large enough, it can hardly locate fault branch, and it can not realize location when there is more than one insulation branch.

\section{The design of a intelligent insulation monitoring device}

On the basis of researching on insulation monitoring methods, a digital on-line insulation monitoring device based on S7-1200 was designed, the design adopts the principle of megameter. 


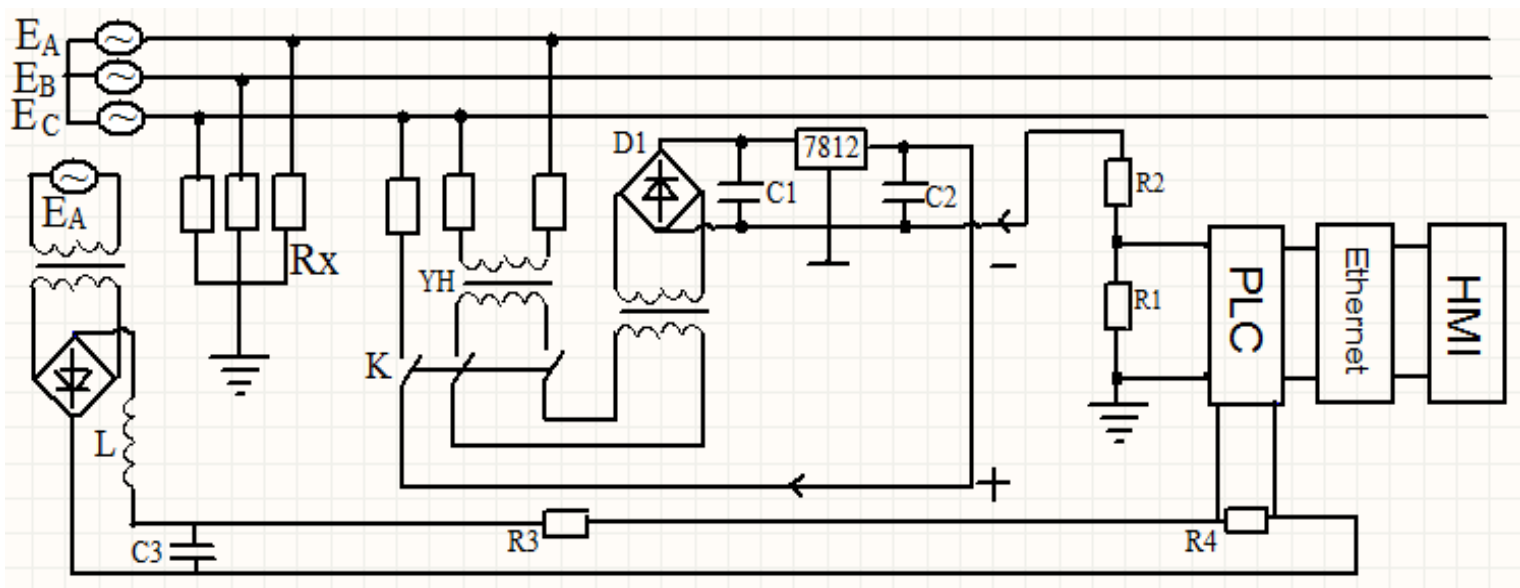

Figure6 the schematic diagram of design on marine insulation monitoring

The traditional megameter include two parts: a measuring circuit which consists of sampling resistance and magneto-electric instrument, and an additional device which provides megameter with power supply. The design reserves the additional device, and mainly improve the measuring circuit. The schematic diagram of the design is shown as figure6, in the diagram, $\mathrm{R}_{1}$ is the sampling resistor of insulation, $R_{2}$ and $R_{3}$ are the current-limiting resistors, $R_{4}$ is the sampling resistor of phase voltage. The additional device provides a stable direct-current power supply whose voltage is $U$, it is in series with $R_{1}, R_{2}$ and insulation resistor $R_{x}$ (the parallel equivalent value of three phase insulation resistors) to form a dc circuit, so the insulation resistor can be calculated out from the schematic diagram:

$$
R_{\mathrm{x}}=\frac{U}{U_{1}} \bullet R_{1}-R_{1}-R_{2}
$$

The insulation resistor $\mathrm{R}_{\mathrm{x}}$ can be accurately calculated by detecting the voltage of resistor $\mathrm{R}_{1}$, and then PLC transmit the calculated insulation data to interface HMI for displaying in real time. At the same time, the design can realize insulation fault phase location by detecting the phase voltage, it is convenient for next accurate insulation fault location. The schematic diagram only gives the detecting circuit of phase A, the working principle of other two phases is identical. Next, take phase A for example to explain the principle of insulation fault location, when insulation fault is detected by the device, if the detecting phase voltage is less than the insulation fault set value, and then PLC will control the buzzer sounding and send a fault signal to HMI to light the indicting lamp of phase A.

The structure and working principle of the design are simple. Setting against to traditional megameter, the device improve the precision of insulation data, and it can realize not only insulation on-line monitoring in real time and digital display, but also history alert data recording and insulation fault phase location, and the insulation can be transmitted to any remote up-computer because of the existence of network cable.

\section{Conclusion}

To sum up in conclusions, nowadays the insulation monitoring of marine power grid is mainly accomplished with megameter or dc superposition method, the insulation fault location mainly adopts double frequency principle, zero sequence current amplitude comparison method. As a sword has two edges, there are some advantages and disadvantages for these methods, in practical applications, it is necessary for users to choose a proper method according to the practical situations of marine power system, or combine these methods to realize the insulation monitoring and fault location.

\section{References}

[1] Wu Zhiliang. Ship power plant and it's automation system[M]. Dalian: the press of Dalian maritime university, 2010 
[2] Yao Donghong, Wang Xinzhi, Liu Lianchao, Zhang Jun. Design of Hot-line insulation monitoring device of dock power cable[J]. Ship electric technology, 2015，35 (7): 57-61

[3] Wang Yong. Research of online insulation monitoring system for power system of ship[M], the master thesis of Huazhong university of science and technology, 2007

[4] Zhuang Jinwu, Xu Guoshun, Qi liansuo, Zhang Chao. Research on application of double frequency principle to insulation fault locating in earth free AC system[J]. Electric power automation equipment, 2003,23 (2): 83-86

[5] Qian wei, Shen Bing, Xu Guoshun, Zheng Gang. An equipment for inspecting the insulation of intermediate frequency net on shipboard[J]. Ship \& ocean engineering, 2008, 37(6):118-121

[6] Zhuang Jinwu, Yang Feng, Shen Bing. Research on fault feeder detection device for shipboard AC system based on current saturation[J]. Relay, 2005,33 (5): 38-41 